University of Wollongong

Research Online

Faculty of Engineering and Information

Faculty of Engineering and Information

Sciences - Papers: Part A

Sciences

$1-1-2013$

\title{
Risk management in liner ship fleet deployment: a joint chance constrained programming model
}

\author{
Tingsong Wang \\ Wuhan University \\ Qiang Meng \\ National University of Singapore \\ Shuaian Wang \\ University of Wollongong, shuaian@uow.edu.au \\ Zhijia Tan \\ Huazhong University of Science \& Technology
}

Follow this and additional works at: https://ro.uow.edu.au/eispapers

Part of the Engineering Commons, and the Science and Technology Studies Commons

Research Online is the open access institutional repository for the University of Wollongong. For further information contact the UOW Library: research-pubs@uow.edu.au 


\title{
Risk management in liner ship fleet deployment: a joint chance constrained programming model
}

\author{
Abstract \\ This paper provides a tangible methodology to deal with the liner ship fleet deployment problem aiming at \\ minimizing the total cost while maintaining a service level under uncertain container demand. The \\ problem is first formulated as a joint chance constrained programming model, and the sample average \\ approximation method and mixed-integer programming are used to deal with it. Finally, a numerical \\ example of a liner shipping network is carried out to verify the applicability of the proposed model and \\ solution algorithm. It is found that the service level has significant effect on the total cost.

\section{Keywords} \\ era2015 \\ Disciplines \\ Engineering | Science and Technology Studies

\section{Publication Details} \\ Wang, T., Meng, Q., Wang, S. \& Tan, Z. (2013). Risk management in liner ship fleet deployment: a joint \\ chance constrained programming model. Transportation Research Part E: Logistics and Transportation \\ Review, 60 1-12.
}




\title{
Risk Management of Uncertain Demand in Liner Ship Fleet Deployment: A Joint Chance Constrained Programming Model
}

\author{
Tingsong Wang ${ }^{1 *}$, Qiang Meng ${ }^{1}$, Shuaian Wang ${ }^{1}$ and Zhijia Tan ${ }^{2}$ \\ ${ }^{1}$ Department of Civil and Environmental Engineering, National University of Singapore, \\ Singapore, 117576 \\ ${ }^{2}$ School of Management, Huazhong University of Science \& Technology \\ China, 430074
}

\begin{abstract}
The liner ship fleet deployment problem with uncertain container demand is one of the risk management issues in liner shipping industry. This paper provides a methodology to deal with this problem, which ensures that the deployed liner ship fleet satisfy the shipping requirement of shippers at least with a predetermined probability. The problem is formulated as a joint chance constrained programming (JCCP) model to minimize the total expected cost incurred in container shipment. As the critical issue of the JCCP model is that the closed forms of the joint chance constraints are analytically intractable, the sample average approximation (SAA) method is used to deal with this issue and a SAA model is then proposed to approximate the JCCP model. Further, the SAA model is equivalently transformed into a mixed-integer linear programming (MIP) model which can be efficiently solved by an optimization solver CPLEX. Finally, a sensitivity analysis is conducted to evaluate the performance of SAA method and a numerical example of a real world liner shipping network provided by a liner shipping company is carried out to show the risk analysis based on the proposed model.
\end{abstract}

Keywords: risk management; liner ship fleet deployment; uncertain demand; joint chance constraint; sample average approximation

\footnotetext{
${ }^{*}$ Corresponding author

E-mail: wangtingsong007@gmail.com(T. Wang)
} 


\section{Introduction}

Liner shipping involves picking up and delivering containerized cargoes (containers) on regularly scheduled shipping routes. Due to its regular and reliable service, liner shipping occupies a dominant proportion of the global shipping market share with $60 \%$ of cargoes by value (Stopford, 2009) and 70\% of containers by volume in terms of TEUs (Twenty-foot Equivalent Units) (UNCTAD, 2011). Consequently, liner shipping is of considerable significance for shipping industry and attracts the attention of researchers in the recent years (see Meng and Wang, 2011a; Wang and Meng, 2012b). As the liner shipping market is intensely competitive, a liner container shipping company has to provide efficient liner shipping service for shippers with the aim of survival and development. Therefore, the liner ship fleet deployment (LSFD) problems that address the assignment of types and numbers of ships to each shipping route at lowest cost in order to effectively utilize and manage these ships are highly concerned about by the liner shipping industry.

Container demand between any two ports of call is a key input of the LSFD problems. Before the actual container demand is realized, decisions of types and numbers of ships assigned to shipping routes have to be made using the estimated container demand. However, some uncontrollable and unpredicted factors such as the cancellation of a shipping contract or the delay in arrival of containers at the port, etc, do exist in practice. As a result, it is almost impossible for the estimated container demand to match the realistic demand precisely. Whatever overestimate or underestimate of the demand, it will lead to a loss for a liner container shipping company. The potential of uncontrollable and unpredicted factors that would result in uncertainty of demand is referred to as a risk faced by liner shipping industry in this paper. Consequently, there is a need to take the risk of uncertain container demand into account in LSFD problems. In practice, the container demand often varies from season to season, and hence the liner container shipping company has to alter its service routes and redeploy ships season by season. Therefore, the research of this paper focuses on the risk management of uncertain demand in LSFD problem over a short-term planning horizon (3 6 months).

\subsection{Literature review}

\subsubsection{Liner ship fleet deployment problems}

Perakis and Jaramillo (1991) made the first step to develop a linear programming model for a 
LSFD problem. However, a flaw in this model is that it unrealistically assumes that the decision variables of number of ships allocated to a shipping route are continuous rather than integers. The same two authors thus built an integer based linear programming model (Jaramillo and Perakis, 1991). By introducing generalized incidence matrices, Cho and Perakis (1996) simplified the expression of mathematical optimization models for LSFD problems in a matrix form. Powell and Perakis (1997) extended the model of Jaramillo and Perakis (1991) by adding the ship lay-up costs to the objective function. Building on their work, Gelareh and Meng (2010) involved ship speed optimization and proposed a nonlinear programming model to determine the optimal ship sailing speed. While this nonlinear programming model can be equivalently reformulated as a linear programming model, the formulation was further improved by Wang et al. (2011). Meng and Wang (2011b) examined a multi-period liner ship fleet planning and deployment problem with known demand in each period. Wang and Meng (2012a) investigated the ship fleet deployment problem with weekly demand and transshipment at any port, and this problem was extended by adding transit time constraints (Meng and Wang, 2012).

\subsubsection{Risk analysis in shipping industry}

It is found that none of the research reviewed above captures the uncertainty of demand in LSFD problems. Studies on network design (e.g., Fagerholt, 1999, 2004; Sambracos et al., 2004; Alvarez, 2009; Karlaftis et al., 2009; Brouer et al., 2011; Jepsen et al., 2011; Reinhardt and Pisinger, 2012) and empty container repositioning (e.g., Song and Dong, 2011; Song and Xu, 2012) have also examined the fixed container demand in liner shipping. Bell et al. (2011), Wang and Meng (2012c) and Qi and Song (2012) have incorporated the uncertainty in the liner service schedules but not investigated the demand uncertainty. The uncertain demand deserves additional research effort (Ronen, 1983, 1993, Christiansen et al., 2004, 2007). To handle demand uncertainty, Meng and Wang (2010) proposed a chance constrained programming approach by which a deterministic LSFD problem was extended to account for the uncertainties. However, this study assumed that all ships have to be emptied at the start of each sailing voyage, which is not consistent with practice. Some other studies focus on risk analysis of currency fluctuation to liner shipping industry (Menachof, 1996), fuel price fluctuation to shipper (Menachof and Dicer, 2001) and default risk in charter market (Adland and Jia, 2008). 


\subsection{Objective and contribution}

The above literature review clearly indicates that the LSFD problem involving container demand uncertainty remains a current research issue with practical significance. The research of this paper focuses on this issue and proposes a joint chance constrained programming (JCCP) model to cope with it. As chance constraints with probability functions in the JCCP model have no closed form, the JCCP model is quite difficult to evaluate. The sample average approximation (SAA) approach is thus used to approximate the JCCP model in this study.

The contribution of this paper is fourfold: First, it contributes to the literature by proposing a realistic LSFD problem with uncertain demand. Second, a JCCP model is developed for the proposed LSFD problem. Unlike Meng and Wang (2010) which defined the level of service for each liner service route, this study examines the demand uncertainty by enforcing a level of service at the network level. This modeling approach not only nests the model of Meng and Wang (2010) as a special case, but also is more practical and relevant as it provides a liner shipping company service information regarding the whole network. Third, an appropriate solution algorithm is proposed to solve the JCCP model. The model proposed by Meng and Wang (2010) can be transformed to a deterministic model because it defines the level of service for each liner service route. However, as we define the level of service at the network level, the mathematical model cannot be transformed to deterministic model directly and its feasible region is non-convex. We successfully apply a sample average approximation approach to address this problem. Fourth, the proposed model and solution algorithm are applied to randomly generated test instances and real-case examples. The results demonstrate that the model and algorithm can analyze risk in the liner ship fleet deployment.

The remainder of this paper is organized as follows: Section 2 presents the LSFD problem with uncertain demand in details. Section 3 develops a JCCP model for the proposed LSFD problem. Section 4 addresses the difficulties in solving the JCCP model and proposes the SAA approach to handle these difficulties. Section 5 uses a numerical example to evaluate the model and solution algorithm proposed in this study. Finally, Section 6 concludes the study and provides recommendations for future work.

\section{Problem Statement}

This section firstly describes a coding scheme for a shipping route, and then addresses the 
container shipment flow, and finally presents the proposed LSFD problem with uncertain demand in details. It is noted that the coding scheme for a shipping route and the concept of container shipment flow have been addressed by Meng and Wang (2011b), but for completeness and the sake of presentation in this paper, they are briefly readdressed here.

\subsection{Shipping route coding scheme}

A shipping route is an itinerary of ship sailing which shows the ports of call on the sail. It is determined by a liner container shipping company and released to shippers for information. Assume that the liner container shipping company operates a heterogeneous fleet of ships on a number of shipping routes, denoted by the set $\mathrm{R}$, to regularly serve a group of ports denoted by the set $\mathrm{P}$. A shipping route $r \in \mathrm{R}$ can be expressed as below according to its ports of call order:

$$
p_{r}^{1} \rightarrow p_{r}^{2} \rightarrow \cdots \rightarrow p_{r}^{m_{r}} \rightarrow p_{r}^{1}
$$

where $p_{r}^{i} \in \mathrm{P} \quad\left(i=1, \cdots, m_{r}\right)$ is the $i^{\text {th }}$ port of call on shipping route $r$ and $m_{r}$ is the number of ports of call on this shipping route. For example, Figure 1 depicts a shipping route which departures from Pusan (PS) port, and sails to call Shanghai (SH) port, Yantian (YT) port, Hong Kong (HK) port, Singapore (SG) port, Yantian (YT) port, and finally returns back to Pusan (PS) port. According to Eq. (1), this shipping route can be coded as below:

$$
p_{r}^{1}(\mathrm{PS}) \rightarrow p_{r}^{2}(\mathrm{SH}) \rightarrow p_{r}^{3}(\mathrm{YT}) \rightarrow p_{r}^{4}(\mathrm{HK}) \rightarrow p_{r}^{5}(\mathrm{SG}) \rightarrow p_{r}^{6}(\mathrm{YT}) \rightarrow p_{r}^{1}(\mathrm{PS})
$$

\subsection{Container shipment flow}

Let $\mathrm{P}_{r}=\left\{p_{r}^{1}, \ldots, p_{r}^{i}, \ldots p_{r}^{m_{r}}\right\}$ be the set of ports called at shipping route $r \in \mathrm{R}$, characterized by $\mathrm{P}=\bigcup_{r \in \mathrm{R}} \mathrm{P}_{r}$, and let $\left(p_{r}^{i}, p_{r}^{j}\right)$ denote the port pair from port $p_{r}^{i}$ to port $p_{r}^{j}$. The set of port pairs having container demand on shipping route $r \in \mathrm{R}$ can be expressed by

$$
\mathrm{M}_{r}=\left\{\left(p_{r}^{i}, p_{r}^{j}\right) \mid i, j=1,2, \cdots, m_{r} ; p_{r}^{i} \neq p_{r}^{j}\right\}
$$

As aforementioned, the container demand of a port pair $\left(p_{r}^{i}, p_{r}^{j}\right) \in \mathrm{M}_{r}$ on shipping route $r \in \mathrm{R}$ is uncertain and denoted by a random variable $\xi^{\left(p_{r}^{i}, p_{r}^{j}\right)}$. A leg $i$ of shipping route $r$ is defined as the voyage from port $p_{r}^{i}$ to port $p_{r}^{i+1}, i=1,2, \cdots, m_{r}-1$, and leg $m_{r}$ stands for the 
voyage from port $p_{r}^{m_{r}}$ to port $p_{r}^{1}$. When a ship sails on leg $l\left(l=1,2, \cdots, m_{r}\right)$ of shipping route $r$, containers on the ship includes those newly loaded at port $p_{r}^{l}$ as well as those loaded at previous ports but still remained on ship, which is referred to as container shipment flow on leg $l$ of shipping route $r$, denoted by $\eta_{l}^{r}$. Therefore, the container shipment flow is an accumulation of containers of some port pairs. Mathematically, it can be expressed as below:

$$
\eta_{l}^{r}=\sum_{\left(p_{r}^{i}, p_{r}^{j}\right) \in \mathrm{M}_{r}} \rho_{l}^{\left(p_{r}^{i}, p_{r}^{j}\right)_{\xi}\left(p_{r}^{i}, p_{r}^{j}\right)}, l=1, \cdots, m_{r} ; \forall r \in \mathrm{R}
$$

where $\rho_{l}^{\left(p_{r}^{i}, p_{r}^{j}\right)}\left(l=1,2, \cdots, m_{r}\right)$ is an incidence parameter which equals 1 if leg $l\left(l=1,2, \cdots, m_{r}\right)$ is contained in a ship's journey of transporting containers from port $p_{r}^{i}$ to port $p_{r}^{j}$ and 0 otherwise $(r \in \mathrm{R})$. Let us take the container shipment flow on leg 6 in Figure 1 to illustrate the Eq. (4). It involves containers of eight port pairs: $\left(p_{r}^{2}, p_{r}^{1}\right),\left(p_{r}^{3}, p_{r}^{1}\right),\left(p_{r}^{4}, p_{r}^{1}\right),\left(p_{r}^{5}, p_{r}^{1}\right)$, $\left(p_{r}^{3}, p_{r}^{2}\right),\left(p_{r}^{4}, p_{r}^{2}\right),\left(p_{r}^{5}, p_{r}^{2}\right)$ and $\left(p_{r}^{5}, p_{r}^{4}\right)$. It is found that the incidence parameters of these eight port pairs on leg 6 equal to 1, and incidence parameters of other port pairs on leg 6 equal to 0 . Therefore, Eq. (4) is satisfied. Let $\eta^{r}=\max _{l=1, \cdots, m_{r}}\left(\eta_{l}^{r}\right), \forall r \in \mathrm{R}$, then it denotes the maximal container shipment flow on shipping route $r$.

\subsection{Liner ship fleet deployment}

As chartering ships through brokers is common in liner shipping, it is thus taken into account in the proposed LSFD problem. Following the fact that the number and types of ships owned or chartered are finite, we assume that there are $K$ types of ships with different size available to the liner container shipping company, denoted by the set $\mathrm{K}=\{1 \cdots k \cdots K\}$, in which $k$ denotes a particular ship type, and let $V_{k}$ be the capacity in terms of TEUs for a particular ship type $k \in \mathrm{K}$, $N_{k}^{\mathrm{MAX}}$ and $N C I_{k}^{\mathrm{MAX}}$ denotes the number of available ships of type $k$ owned and chartered by the liner container shipping company, respectively.

The total costs incurred in liner shipping typically consist of four blocks (Stopford, 2009): operating costs, voyage costs, capital costs and periodic maintenance. Operating costs refer to the day-to-day ongoing expenses of running the ship, including manning cost, stores and 
consumables, insurance and administration cost (excluding fuel, which is included in voyage costs), together with an allowance for day-to-day routine repairs and maintenance. In summary, the operating cost structure depends on the size and nationality of the crew, maintenance policy and the age and insured value of the ship, and the administrative efficiency of the owner. The voyage costs are defined as the variable costs incurred in undertaking a particular voyage. The main items are fuel costs, port dues, tugs and pilotage and canal charges. Capital costs mainly refer to the investment of purchasing or chartering ships. Here in this paper, purchasing ships is excluded because chartering ships is a better choice in a short-term planning horizon for the company from the view point of economics, if any. Periodic maintenance is a provision set aside to cover the cost of interim dry-docking and special surveys. The ship must be dry-docked every two years and every four years must have a special survey, approving its seaworthiness. Since the planning horizon considered in this paper is less than two years, the periodic maintenance is excluded. Therefore, the total costs of ships incurred in the LSFD problem contain three components: operating costs, voyage costs and chartering costs.

Before proceeding to the modeling of the LSFD problem, we finally completely state it as follows: determine the number of ships of each type to charter in/out, the type and number of ships to deploy on each shipping route, and the number of voyages to be completed on each shipping route, to provide shipping service for shippers while minimizing the total costs.

\section{Model Development}

According to the statement of LSFD problem above, there are three types of decision variables involved:

$n_{k r}^{\text {TOTAL }}:$ number of ships (the sum of owned and chartered in ships) of type $k(k \in \mathrm{K})$ assigned on route $r(r \in \mathrm{R})$

$n_{k}^{\mathrm{IN}}: \quad$ number of chartered in ships of type $k(k \in \mathrm{K})$

$x_{k r}: \quad$ number of voyages completed by ships of type $k(k \in \mathrm{K})$ on route $r(r \in \mathrm{R})$

\subsection{Costs function}

We firstly compute the operating costs. Let $c_{k}^{\text {operate }}$ (USD/day) denote the operating costs of a ship of type $k \in \mathrm{K}, T$ (days) denote the length of the short-term planning horizon, then the 
operating costs of all ships in the planning horizon can be computed by $\sum_{r \in \mathrm{R}} \sum_{k \in \mathrm{K}} n_{k r}^{\mathrm{TOTAL}} c_{k}^{\text {operate }} T$. As for voyage costs of all ships, it equals to $\sum_{r \in \mathrm{R}} \sum_{k \in \mathrm{K}} c_{k r}^{\text {voyage }} X_{k r}$, where $c_{k r}^{\text {voyage }}$ (USD/voyage) denote the voyage costs of a ship of type $k \in \mathrm{K}$ on shipping route $r \in \mathrm{R}$. Let $c_{k}^{\mathrm{IN}}$ (USD/day) denote the daily cost of chartering in a ship of type $k \in \mathrm{K}$ for the planning horizon, then the total chartering costs can be computed by $\sum_{k \in \mathrm{K}} n_{k}^{\mathrm{IN}} C_{k}^{\mathrm{IN}} T$. For the sake of presentation, we let $\mathbf{x}$ be the vector of all decision variables, namely, $\mathbf{x}=\left(n_{k r}^{\mathrm{TOTAL}}, n_{k}^{\mathrm{IN}}, x_{k r} \mid k \in \mathrm{K}, r \in \mathrm{R}\right)$. Therefore, the cost function with respect to the decision vector $\mathbf{x}$ of the proposed LSFD problem, denoted by $T C(\mathbf{x})$, equals to:

$$
T C(\mathbf{x})=\sum_{r \in \mathrm{R}} \sum_{k \in \mathrm{K}} n_{k r}^{\mathrm{TOTAL}} c_{k}^{\text {operate }} T+\sum_{r \in \mathrm{R}} \sum_{k \in \mathrm{K}} c_{k r}^{\text {voyage }} x_{k r}+\sum_{k \in \mathrm{K}} n_{k}^{\mathrm{IN}} c_{k}^{\mathrm{IN}} T
$$

\subsection{Risk management of uncertain demand}

As aforementioned, the container shipment demand taken into account in this research is uncertain, and such a consideration of uncertainty makes the LSFD problem more realistic. However, it results in a new issue: there is almost no decision which would definitely exclude later constraint violation caused by unexpected random effects. In other words, once the decisions in LSFD problem are determined, the fleet of ships may face such a risk that it is unable to fully meet the pickups and deliveries requirement for its customers, even though the expected container shipment flow along the shipping route do not exceed the fleet capacity. Since such a case is hardly avoidable, the liner container shipping company intends to control its possibility at a low level. In order to reflect the intention, the probability theory is introduced. Let $V_{k}$ denote the size in terms of TEUs of a ship of type $k$, then that the liner container shipping company can satisfy the customers' shipping requirement with a probability of $1-\alpha$ can be formulated as the following probabilistic form, which is termed as a chance constraint:

$$
\operatorname{Pr}\left(\sum_{k \in \mathrm{K}} x_{k r} V_{k} \geq \eta^{r}, \forall r \in \mathrm{R}\right) \geq 1-\alpha
$$

where the item $\sum_{k \in \mathrm{K}} x_{k r} V_{k}$ denotes the transportation capacity of ships deployed on shipping route $r$, $\eta^{r}$ denotes the maximal container shipment flow on shipping route $r$ as defined before, $\alpha \in(0,1)$ is called confidence parameter in a chance constrained programming model. The 
probability $1-\alpha$ can be regarded as a level of service that the company can maintain. For those unmet cargoes, we regarded them lost.

\subsection{Joint chance constrained programming model}

With the consideration of demand uncertainty for LSFD problem in this paper, there is a need to build a mathematical model to handle the risk management of uncertain demand. Based on the description in Section 3.2, the proposed LSFD problem with uncertain demand aims to maintain a level of service on each ship route for customers while minimizing the total costs. It is formulated as a joint chance constrained programming model, named JCCP-1:

[JCCP-1]

$$
z_{\alpha}^{*}=\min _{\mathbf{x}} T C(\mathbf{x})
$$

subject to

$$
\begin{gathered}
\sum_{r \in \mathrm{R}} n_{k r}^{\mathrm{TOTAL}} \leq N_{k}^{\mathrm{MAX}}+n_{k}^{\mathrm{IN}}, \forall k \in \mathrm{K} \\
n_{k}^{\mathrm{IN}} \leq N C I_{k}^{\mathrm{MAX}}, \forall k \in \mathrm{K} \\
x_{k r} \leq n_{k r}^{\mathrm{TOTAL}} \times\left\lfloor\frac{T}{t_{k r}}\right\rfloor, \forall r \in \mathrm{R}, k \in \mathrm{K} \\
\sum_{k \in \mathrm{K}} x_{k r} \geq N_{r}, \forall r \in \mathrm{R} \\
\operatorname{Pr}\left(\sum_{k \in \mathrm{K}} x_{k r} V_{k} \geq \eta^{r}, \forall r \in \mathrm{R}\right) \geq 1-\alpha \\
n_{k r}^{\text {TOTAL }}, n_{k}^{\mathrm{IN}}, \quad x_{k r} \in \square^{+} \bigcup\{0\}, \forall k \in \mathrm{K}, \forall r \in \mathrm{R}
\end{gathered}
$$

where $z_{\alpha}^{*}$ denotes the value of the objective function in Eq. (7), $t_{k r}$ is the voyage time of a ship of type $k$ on a particular shipping route $r$ (in days), $N_{r}$ is the minimal number of voyages required on shipping route $r$ during the planning horizon in order to maintain a given liner shipping service frequency.

Eq. (7) is the objective function of the JCCP-1 model. The set of constraints (8) ensure that the total number of ships used in the fleet, including those owned and those chartered in, does not exceed the number of available ships. The set of constraints (9) indicates that the number of chartered in ships is finite and does not exceed the number of available ships. The right-hand side of constraints (10) gives the maximal number of voyages that ships deployed on route $r$ can 
complete in the planning horizon, where $\lfloor a\rfloor$ denotes the maximum integer not greater than $a$. Therefore, the set of constraints (10) is the upper bound for the decision variables $x_{k r}$. The constraints given by Eq. (11) require that ships deployed on shipping route $r$ have to complete at least $N_{r}$ voyages in order to maintain the given liner shipping frequency. For example, if a weekly shipping service is required on shipping route $r$ during a planning horizon of six months, then $N_{r}=26$. Constraint (12) is a joint chance constraint which ensures that the ships on all shipping routes can at least satisfy customers' requirement with a probability of $1-\alpha$.

Constraint (12) can be rewritten in another form. Let $G^{r}\left(\hat{\mathbf{x}}, \eta^{r}\right):=\eta^{r}-\sum_{k \in \mathrm{K}} x_{k r} V_{k}$, (这里为什么 用 $\mathrm{x}$ 尖而不用 $\mathrm{x}$ 了? ) where $\hat{\mathbf{x}}=\left(x_{k r} \mid k \in \mathrm{K}, r \in \mathrm{R}\right)$, and let $G(\hat{\mathbf{x}}, \eta):=\max _{\forall r \in \mathrm{R}} G^{r}\left(\hat{\mathbf{x}}, \eta^{r}\right)$, we define the probability functions $p(\hat{\mathbf{x}}):=\operatorname{Pr}(G(\hat{\mathbf{x}}, \eta)>0)$, then constraint (12) is equivalent to the equation below:

$$
p(\hat{\mathbf{x}}) \leq \alpha
$$

Therefore, we have another JCCP model with a joint chance constraint (12) replaced by (14), named JCCP-2:

[JCCP-2]

$$
z_{\alpha}^{*}=\min _{\mathbf{x}} T C(\mathbf{x})
$$

subject to $(8) \sim(11),(13)$ and (14)

\section{Solution Algorithm}

Chance constrained programming (CCP) was first introduced and studied by Charnes et al. (1958) more than 50 years ago. Since then, it has been studied extensively in the stochastic optimization literature (Prékopa, 2003). However, this problem is still considered as challenging because of the two major extreme difficulties to solve it: one is that the feasible region defined by a probabilistic constraint in $\mathrm{CCP}$ is generally not convex; another is that the chance constraints generally have no closed forms and are typically difficult to evaluate (Miller and Wagner, 1965). To address these difficulties, different approaches have been proposed in the stochastic optimization literature and can be classified into two somewhat different directions: one is to employ convex approximations of chance constraints (Ben-Tal and Nemirovski, 2000; Hong et al., 2011), another is to discretize the probability distribution and use Monte Carlo simulation to 
approximate the obtained problem (Dentcheva et al., 2000; Pagnoncelli et al., 2009). The convex approximation approaches usually require that the decision variables are continuous; however, the decision variables involved in the proposed JCCP models (JCCP-1 and JCCP-2) are restricted to be integers, the convex approximation approaches are thus not applicable for our problem. Therefore, the approach in the second direction, specifically, the Sample Average Approximation (SAA) approach (Atlason et al., 2008; Luedtke and Ahmed, 2008), is then used to seek approximation for the proposed JCCP models.

\subsection{Sample average approximation}

The theoretical background of SAA approach is based on the Law of Large Numbers theory which indicates that the probability of an event occurrence can be approximated by the frequency of the events that occur in number of trials (say $S$ trials). Let $\xi_{1}^{\left(p_{r}^{i}, p_{r}^{j}\right)} \ldots \xi_{S}^{\left(p_{r}^{i}, p_{r}^{j}\right)}$ be an independent Monte Carlo sample of $S$ realizations of the random variable $\xi^{\left(p_{r}^{i}, p_{r}^{j}\right)}$, we then obtain $S$ realization of the random vector $\eta$, denoted by $\eta_{1}, \ldots, \eta_{S}$, and let $l_{(0, \infty)}: R \rightarrow R$ be the indicator function of $(0, \infty)$, i.e.,

$$
I_{(0, \infty)}(y):= \begin{cases}1, & \text { if } y>0 \\ 0, & \text { if } y \leq 0\end{cases}
$$

Then, the sample version of the probability function $p_{r}(\hat{\mathbf{x}})$ is defined to be

$$
p^{S}(\hat{\mathbf{x}})=S^{-1} \sum_{i=1}^{S} 11_{(0, \infty)}\left(G\left(\hat{\mathbf{x}}, \eta_{i}\right)\right)
$$

That is, $p^{S}(\hat{\mathbf{x}})$ is equal to the proportion of times that $G\left(\hat{\mathbf{x}}, \eta_{i}\right)>0$. The constraint (14) is then replaced by

$$
p^{S}(\hat{\mathbf{x}}) \leq \beta
$$

where $\beta \in(0,1)$ is a confidence parameter and can be different from the original one $\alpha$ (Luedtke and Ahmed, 2008). Finally, the sample version of the JCCP-2 model with a joint chance constraint (14) is named SAA- $\beta$ model and defined as

$$
[\mathrm{SAA}-\beta] \quad \hat{\mathrm{Z}}_{\beta}^{S}=\min _{\mathbf{x}} T C(\mathbf{x})
$$

subject to (8) (11), (13) and (18). 


\subsection{Solving the sample average approximation problem}

Though the joint chance constraint (12) is handled by using the SAA approach, shown in Eq.(18), and the true problem (15) is approximated by the SAA problem (19), the problem (19) is still hard to solve because of the complexity of constraint (18). To solve the sample approximation problem (19), we rewrite it as a mixed-integer program (MIP) with one binary variable $\vartheta_{i}(i=1, \ldots, S)$ for each sample point

[MIP]

$$
\hat{z}_{\beta}^{S}=\min _{\mathbf{x}} T C(\mathbf{x})
$$

Subject to (8) (11), (13), and

$$
\begin{gathered}
\vartheta_{i} \eta_{i}^{r}+\sum_{k \in \mathrm{K}} x_{k r} V_{k} \geq \eta_{i}^{r}, \forall i=1, \ldots, S ; \forall r \in \mathrm{R} \\
\sum_{i=1}^{S} \vartheta_{i} \leq S \beta \\
\vartheta_{i} \in\{0,1\}^{S}
\end{gathered}
$$

Proposition: The Problems (19) and (20) are equivalent.

Proof: Let $\left(\mathbf{x}, \vartheta_{1}, \ldots, \vartheta_{S}\right)$ be feasible solution for problem (20). For each $i=1, \ldots, S$, from constraints (21), we can deduce that if $\forall r \in \mathrm{R}, \sum_{k \in \mathrm{K}} x_{k r} V_{k} \geq \eta_{i}^{r}$, then $\vartheta_{i}=0$ or $\vartheta_{i}=1$, and we have $11_{(0, \infty)}\left(G\left(\hat{\mathbf{x}}, \eta_{i}\right)\right)=0$; if $\exists r \in \mathrm{R}, \sum_{k \in \mathrm{K}} x_{k r} V_{k} \geq \eta_{i}^{r}$, then $\vartheta_{i}=1$ and $11_{(0, \infty)}\left(G\left(\hat{\mathbf{x}}, \eta_{i}\right)\right)=1$; if $\forall r \in \mathrm{R}, \sum_{k \in \mathrm{K}} x_{k r} V_{k} \leq \eta_{i}^{r}$, then $\vartheta_{i}=1$ and $11_{(0, \infty)}\left(G\left(\hat{\mathbf{x}}, \eta_{i}\right)\right)=1$. Therefore, $\vartheta_{i} \geq 11_{(0, \infty)}\left(G\left(\hat{\mathbf{x}}, \eta_{i}\right)\right)$.

Accordingly, from constraint (22), we have $\beta \geq S^{-1} \sum_{i=1}^{S} \vartheta_{i} \geq S^{-1} \sum_{i=1}^{S} 11_{(0, \infty)}\left(G\left(\hat{\mathbf{x}}, \eta_{i}\right)\right)=p^{S}(\hat{\mathbf{x}})$. Thus, $\mathbf{x}$ is feasible to (19) and has the same objective value as in (20). Conversely, let $\mathbf{x}$ be a feasible solution for (19), and define $\vartheta_{i}=11_{(0, \infty)}\left(G\left(\hat{\mathbf{x}}, \eta_{i}\right)\right)$. For each $i=1, \ldots, S$, if $G\left(\hat{\mathbf{x}}, \eta_{i}\right) \leq 0$, then $\vartheta_{i}=0$ and $\sum_{k \in \mathrm{K}} x_{k r} V_{k}>\eta_{i}^{r}, \forall r \in \mathrm{R}$, therefore constraint (21) holds; if $G\left(\hat{\mathbf{x}}, \eta_{i}\right)>0$, then $\vartheta_{i}=1$

and constraint (21) holds as well. As for constraint (22), we have $\sum_{i=1}^{S} \vartheta_{i}=\sum_{i=1}^{S} 11_{(0, \infty)}\left(G\left(\hat{\mathbf{x}}, \eta_{i}\right)\right) \leq S \beta$.

Therefore, we have that $\left(\mathbf{x}, \vartheta_{1}, \ldots, \vartheta_{S}\right)$ is feasible for problem (20) with the same objective value. The proposition is proved. 
Since the SAA- $\beta$ model is an MIP, the optimization solver, CPLEX, can be employed to solve it. Let $\mathbf{X}_{\alpha}$ and $\mathbf{X}_{\beta}^{S}$ denote the set of optimal solutions to the true problem (i.e. JCCP-2 model) and the SAA problem (i.e. SAA- $\beta$ model), respectively. It has been proved that $\hat{z}_{\beta}^{S}$ and $\mathbf{X}_{\beta}^{S}$ converge w.p. 1 to their counterparts of the true problem (i.e. $Z_{\alpha}^{*}$ and $\mathbf{X}_{\alpha}$ ) exponentially fast as $S$ increases under mild regularity conditions (Pagnoncelli et al., 2009).

\subsection{Lower bound}

Increasing the feasible set of an optimization problem aiming at minimizing the value of an objective function may result in decreasing of the optimal objective function value of the problem. Therefore, if we increase the value of $\alpha$ in JCCP-2, then $z_{\alpha}^{*}$ may decrease. In other words, we can obtain a lower bound of JCCP-2 by increasing the value of $\alpha$. However, solving JCCP-2 is extremely difficult which indicates that it is hard for us to obtain the lower bound by solving JCCP-2 with an enlarged $\alpha$. Since the SAA- $\beta$ model is an approximation of the proposed JCCP-2 model, we can expect that the objective function value of the SAA- $\beta$ model in which $\beta>\alpha$, denoted by $\hat{z}_{\beta^{L}}^{S}$, is a lower bound of $z_{\alpha}^{*}$ with some significance level. This expectation has been mathematically proved in Theorem 3 of Luedtke and Ahmed (2008), and accordingly,

the sample size, $S$, to ensure that $\hat{z}_{\beta^{L}}^{S} \leq z_{\alpha}^{*}$ with probability at least $1-\delta$, where $\delta \in(0,1)$, can be estimated by :

$$
S \geq \frac{1}{2(\beta-\alpha)^{2}} \ln \left(\frac{1}{\delta}\right)
$$

\subsection{Verification of solution feasibility}

The above section shows that solving SAA- $\beta$ model in which $\beta^{L}>\alpha$ yields a lower bound of JCCP-2 model with some probability. Contrarily, solving SAA- $\beta$ model with $\beta^{U}<\alpha$ might produce feasible solutions to JCCP-2 model. In other words, it yields an upper bound with some probability, denoted by $\hat{z}_{\beta^{U}}^{S}$. For a given candidate point $\mathbf{x}^{*} \in \mathbf{X}_{\beta^{U}}^{S}$, namely an optimal solution to SAA- $\beta$ model in which $\beta^{U}<\alpha$, we would like to validate its quality as a solution to JCCP-2 
model. For that we need to estimate the probability $p\left(\hat{\mathbf{x}}^{*}\right)$. We proceed the verification by employing again the Monte Carlo sampling techniques. Generate a sample $\xi_{1}^{\left(p_{r}^{i}, p_{r}^{j}\right)} \ldots \xi_{S^{\prime}}^{\left(p_{r}^{i}, p_{r}^{j}\right)}$ with $S^{\prime}$ realizations of the random vector $\eta$. The sample is generated independently of the random procedure which produced the candidate solution $\mathbf{x}^{*}$. Estimate $p\left(\hat{\mathbf{x}}^{*}\right)$ by $p^{S^{\prime}}\left(\hat{\mathbf{x}}^{*}\right)$ because the estimator $p^{S^{\prime}}\left(\hat{\mathbf{x}}^{*}\right)$ is unbiased. It is noted that we can use a very large sample since there is no need to solve any optimization problem here. If $p^{S^{\prime}}\left(\hat{\mathbf{x}}^{*}\right) \leq \alpha$, then $\mathbf{x}^{*}$ is a feasible solution.

Otherwise we choose another smaller $\beta^{L}$, obtain a new solution $\mathbf{x}^{*}$, and check its feasibility. This procedure is repeated until a feasible solution is obtained. It should be mentioned that our computational experiments actually demonstrate that a feasible solution is generally obtained in the first iteration.

\section{Computational Results}

In this section, we firstly conduct a sensitivity analysis of SAA parameters through a preliminary experiment with small scales, in order to choose suitable values of SAA parameters taking into account the trade-off between the quality of the solution obtained for the experiment and the computational effort needed to solve it. With these chosen parameters, we then illustrate the applicability of the proposed model and conduct risk management on a real-world shipping network. The solution algorithm is implemented in a programming language Lua (v5.1) coded in Microcity (http://microcity.sourceforge.net) and the SAA problems are solved by CPLEX (v12.1). All computations are carried out on a desktop personal computer with Intel (R) Core (TM) 2 CPU 1.86 GHz and 2.0 GB of RAM under Microsoft Windows 7.

\subsection{Sensitivity analysis of SAA parameters}

From the above description of SAA approach, it is found that for a JCCP-2 problem with a given confidence parameter, $\alpha$, the parameters, $\beta, \delta, S$ and $S^{\prime}$ need to be determined in the SAA approach. Therefore, the sensitivity analysis of SAA parameters focuses on $\beta, \delta$ and $S$, and it is implemented like this: we firstly test a number of sets of these SAA parameters, the results are shown in Table 1; and then evaluate the performance of the approach with these tested SAA parameters in order to choose the best one. 
We set three different values of $\alpha$, shown in the first column of Table 1. For each value of $\alpha$, five sets of parameters, $\beta, \delta, S$ and $S^{\prime}$, are tested. The values of $S$ in the fifth column satisfy Eq. (24). The relative gap between lower bound and upper bound is computed by $\frac{\hat{z}_{\beta^{U}}^{S}-\hat{z}_{\beta^{L}}^{S}}{\hat{z}_{\beta^{L}}^{S}} \times 100 \%$, shown in Column 7 . The computational time is listed in the last column of Table 1.

As can be seen from Table 1, for each $\alpha$, the relative gap generally increases with the interval between $\beta^{L}$ and $\beta^{U}$. The rationale behind this trend is that when $\beta^{L}$ increases, the feasible set increases as well, which results in that the lower bound $\hat{z}_{\beta^{L}}^{S}$ may decrease. Similarly, the upper bound $\hat{z}_{\beta^{U}}^{S}$ may increase when $\beta^{U}$ decreases. Therefore, it makes the relative gap enlarge for an increasing interval between $\beta^{L}$ and $\beta^{U}$. However, an exception in Table 1 is that the first relative gap in Column 8 for $\alpha=0.05$ is $0.85 \%$, larger than the second one, $0.73 \%$. It is possible for this exception because the JCCP models (or SAA models) involve uncertain parameters and their values are generated randomly. The randomness of parameters may make such an exception occur. Additionally, all of the values of $p^{S^{\prime}}\left(\hat{\mathbf{x}}^{*}\right)$ in Column 8 are less than the corresponding value of $\alpha$, which indicates that the values set for $\beta^{U}$ and $S^{\prime}$ are effective to yield a feasible solution.

\subsection{Real-world case study}

\subsubsection{Dataset description}

The real-world liner shipping network provided by OOCL-a global liner container shipping company with headquarters in Hong Kong (http://www.oocl.com), consists of 36 ports and serves 390 O-D port pairs, as shown in Figure 2. The ports of call and distance of each leg of each shipping route is shown in Table 2. It is assumed that the short-term planning horizon in this numerical example is six months. The relevant ship data are presented in Table 3 , including ship size and type, daily operating cost, etc. The daily operating cost as a function of ship size is estimated using the following linear regression equation, established by Shintani et al. (2007):

$$
\text { daily operating cost }=6.54 \times \text { ship size }(\mathrm{TEU})+1422.5
$$


We assume that the uncertain parameters of container demand in the LSFD test experiment follow log-normal distributions, i.e. $\xi^{\left(p_{r}^{i}, p_{r}^{j}\right)} \square \ln N\left(\mu^{\left(p_{r}^{i}, p_{r}^{j}\right)}, \sigma^{\left(p_{r}^{i}, p_{r}^{j}\right)^{2}}\right)$, to generate the demands because log-normal distributions were well suited for modeling economic stochastic variables such as demands (Kamath and Pakkala, 2002). The ratio $\sigma^{\left(p_{r}^{i}, p_{r}^{j}\right)} / \mu^{\left(p_{r}^{i}, p_{r}^{j}\right)}$ is assumed to be the same for all port pairs, for the sake of presentation, denoted by $\lambda$. Assuming that $\alpha=0.10$, we set $\beta^{L}=0.12, \beta^{U}=0.075, \delta=0.095, S=3000$ and $S^{\prime}=10000$.

\subsubsection{Risk analysis of uncertain demand}

The variance of uncertain container demand can be regarded as representing the risk of shipping market. In the case when $\sigma^{\left(p_{r}^{i}, p_{r}^{j}\right)}=0$, the shipping market can be thought of as non-risky, and the container demand can be predicted precisely. In the case when $\sigma^{\left(p_{r}^{i}, p_{r}^{j}\right)}>0$, it indicates that there is risk in shipping market, and when $\sigma^{\left(p_{r}^{i}, p_{r}^{j}\right)}$ increases, it means that the risk increases as well. In order to study the effect of variance on the cost that the liner shipping company need to maintain a given level of service, namely the objective function value of the JCCP model, we vary the ratio, $\lambda$, from 0 to 0.5 with increments of 0.05 and show the trend in the cost as $\lambda$ changes in Figure 3. As can be seen from that the trend generally increases with $\lambda$ increases. It shows that the variability of the uncertain parameters has a significant effect on the solutions.

\section{Conclusion}

In this study, a realistic LSFP problem with container demand uncertainty encountered by a liner shipping company has been considered. A concept of level of service is introduced in this problem to deal with the risk management of uncertain demand and a JCCP model is proposed for it. It is possible to adapt the methodology of model formulation of the problem to other planning problems that involve uncertain demand, such as supply chain system design. The challenge to solve the JCCP model is that the joint chance constraints generally have no closed forms and thus are hard to evaluate. To effectively solve the proposed JCCP model, we firstly applied sample average approximation approach and then proposed a SAA model to approximate the model; further, we equivalently transformed the SAA model into a MIP model and solved it by using 
CPLEX solver. A sensitivity analysis of SAA parameters through a preliminary experiment was firstly conducted and then the proposed model and solution algorithm were tested using a real world liner shipping network. The gaps between the lower and upper bounds are small, which indicates that the solution scheme is effective. It is also found that the variability of the uncertain parameters has a significant effect on the solutions. We believe that the model provides a credible and effective methodology for the real world LSFD problem in an uncertain environment.

\section{Acknowledgements}

This study is supported by the research grants WBS No. R-264-000-244-720 and WBS No. R-302-000-014-720 from the Neptune Orient Lines (NOL) Fellowship Programme of Singapore.

\section{REFERENCES}

Adland, R., and Jia, H. (2008). Charter market default risk: A conceptual approach. Transportation Research Part E , 44 (1), 152-163.

Altason, J., Epelman, M., and Henderson, G. (2008). Optimizing call center staffing using simulation and analytic center cutting-plane methods. Management Science, 54 (2), 295-309.

Alvarez, J.F. (2009). Joint routing and deployment of a fleet of container vessels. Maritime Economics \& Logistics, 11, 186-208.

Bell, M.G.H., Liu, X., Angeloudis, P., Fonzone, A., Hosseinloo, S.H. (2011). A frequency-based maritime container assignment model. Transportation Research Part B, 45, 1152-1161.

Ben-Tal, A., and Nemirovski, A. (2000). Robust solutions of linear programming problems contaminated with uncertain data. Mathematical Programming , 88 (3), 411-424.

Brouer, B.D., Pisinger, D., Spoorendonk, S. (2011). Liner shipping cargo allocation with repositioning of empty containers. INFOR, 49(2), 109-124.

Charnes, A., Cooper, W., and Symonds, G. (1958). Cost horizons and certainty equivalents: An approach to stochastic programming of heating oil. Management Science , 4 (3), 235-263.

Cho, S., and Perakis, A. (1996). Optimal liner fleet routing strategies. Maritime Policy and Management, 23, 249-259.

Christiansen, M., Fagerholt, K., Ronen, D. (2004). Ship routing and scheduling: status and perspectives. Transportation Science, 38(1), 1-18.

Christiansen, M., Fagerholt, K., Nygreen, B., Ronen, D. (2007). Maritime transportation. In: Barnhart, C. and Laporte, G. (Eds.), Handbook in OR \& MS, Volume 14. Elsevier, 189-284. 
Dentcheva, D., Prékopa, A., and Ruszczyński, A. (2000). Concavity and efficient points of discrete distribution in probabilistic programming. Mathematical Programming, 89, 55-77.

Fagerholt, K. (1999). Optimal fleet design in a ship routing problem. International Transactions in Operational Research, 6, 453-464.

Fagerholt, K. (2004). Designing optimal routes in a liner shipping problem. Maritime Policy and Management, 31(4), 259-268.

Gelareh, S., and Meng, Q. (2010). A novel modeling approach for the fleet deployment problem within a short-term planning horizon. Transportation Research Part E , 46 (1), 76-89.

Hong, L., Yang, Y., and Zhang, L. (2011). Sequential convex approximation to joint chance constrained programs: A Monte Carlo approach. Operations Research , 59 (3), 617-630.

Jaramillo, D. I., and Perakis, A. (1991). Fleet deployment optimization for liner shipping Part 2. Implementation and results. Maritime Policy and Management, 18 (3), 235-262.

Jepsen, M.K., Løfstedt, B., Plum, C.E.M., Pisinger, D., Sigurd, M.M. (2011). A path based model for a green liner shipping network design problem. Proceedings of the International MultiConference of Engineers and Computer Scientists 2011.

Kamath, K., and Pakkala, T. (2002). A Bayesian approach to a dynamic inventory model under an unknown demand distribution. Computers and Operations Research , 29 (4), 403-422.

Karlaftis, M.G., Kepaptsoglou, K., Sambracos, E. (2009). Containership routing with time deadlines and simultaneous deliveries and pick-ups. Transportation Research Part E, 45, 210-221.

Luedtke, L., and Ahmed, S. (2008). A sample approximation approach for optimization with probalistic constraints. SIAM Journal on Optimization , 19 (2), 674-699.

Menachof, D. (1996). Risk management methods for the liner shipping industry: the response to customer service demands for simplified tariffs. Journal of Business Logistics , 17 (1), 259-291.

Menachof, D., and Dicer, G. (2001). Risk management methods for the liner shipping industry: the case of the Bunker Adjustment Factor. Maritime Policy and Management , 28 (2), 141-155.

Meng, Q., and Wang, S. (2011a). Optimal operating strategy for a long-haul liner service route. European Journal of Operational Research , 215 (1), 105-114. 
Meng, Q., and Wang, S. (2012). Liner ship fleet deployment with week-dependent container shipment demand. European Journal of Operational Research , In press.

Meng, Q., and Wang, T. (2010). A chance constrained programming model for short-term liner ship fleet planning problems. Maritime Policy and Management, 37 (4), 329-346.

Meng, Q., and Wang, T. (2011b). A scenario-based dynamic programming model for multi-period liner ship fleet planning. Transportation Research Part E , 47 (4), 401-413.

Miller, L., and Wagner, H. (1965). Chance-constrained programming with joint constraints. Operations Research , 13 (6), 930-945.

Pagnoncelli, B., Ahmed, S., and Shapiro, A. (2009). Sample average approximation method for chance constrained programming: theory and applications. Journal of Optimization Theory and Applications , 142 (2), 399-416.

Perakis, A., and Jaramillo, D. I. (1991). Fleet deployment optimization for liner shipping Part 1. Background, problem formulation and solution approaches. Maritime Policy and Management, 18 (3), 183-200.

Powell, B., and Perakis, A. (1997). Fleet deployment optimization for liner shipping: an integer programming model. Maritime Policy and Management , 24 (2), 183-192.

Prékopa, A. (2003). Probability programming. In A. Ruszczyński, \& A. Shapiro, Stochastic Programming, in: Handbook in OR \& MS (Vol. 10, pp. 267-352). Amsterdam: Elsevier.

Qi, X., Song, D.P. (2012). Minimizing fuel emissions by optimizing vessel schedules in liner shipping with uncertain port times. Transportation Research Part E, 48, 863-880.

Reinhardt, L.B., Pisinger, D. (2012). A branch and cut algorithm for the container shipping network design problem. Flexible Services and Manufacturing Journal, doi: 10.1007/s10696-011-9105-4.

Ronen, D. (1983). Cargo ships routing and scheduling: Survey of models and problems. European Journal of Operational Research, 12, 119-126.

Ronen, D. (1993). Ship scheduling: The last decade. European Journal of Operational Research, $71,325-333$.

Sambracos, E., Paravantis, J.A., Tarantilis, C.D., Kiranoudis, C.T. (2004). Dispatching of small containers via coastal freight liners: The case of the Aegean Sea. European Journal of Operational Research, 152, 365-381. 
Shintani, K., Imai, A., Nishimura, E., and Papadimitriou, S. (2007). The container shipping network design problem with empty container repositioning. Transportation Research Part E, $43(1), 39-59$.

Song, D.P., Dong, J.X. (2011). Effectiveness of an empty container repositioning policy with flexible destination ports. Transport Policy, 18(1), 92-101.

Song, D.P. and Xu, J.J. (2012). An operational activity-based method to estimate $\mathrm{CO}_{2}$ emissions from container shipping considering empty container repositioning, Transportation Research Part D, 17, 91-96.

Stopford, M. (2009). Maritime Economics (third ed.). London: Routledge.

UNCTAD, 2011. Review of Maritime Transportation 2011. Paper presented at the United Nations Conference on Trade and Development. New York and Geneva, USA. http://www.unctad.org/templates/webflyer.asp?docid=15876\&intItemID=2068\&lang=1\&mod $\mathrm{e}=$ downloads.

Wang, S., and Meng, Q. (2012a). Liner ship fleet deployment with container transshipment operations. Transportation Research Part E , 48 (2), 470-484.

Wang, S., and Meng, Q. (2012b). Sailing speed optimization for container ships in a liner shipping network. Transportation Research Part E , 48 (3), 701-714.

Wang, S., and Meng, Q. (2012c). Liner ship route schedule design with sea contingency time and port time uncertainty. Transportation Research Part B , 46 (5), 615-633.

Wang, S., Wang, T., and Meng, Q. (2011). A note on liner ship fleet deployment. Flexible Services and Manufacturing Journal , 23 (4), 422-430. 


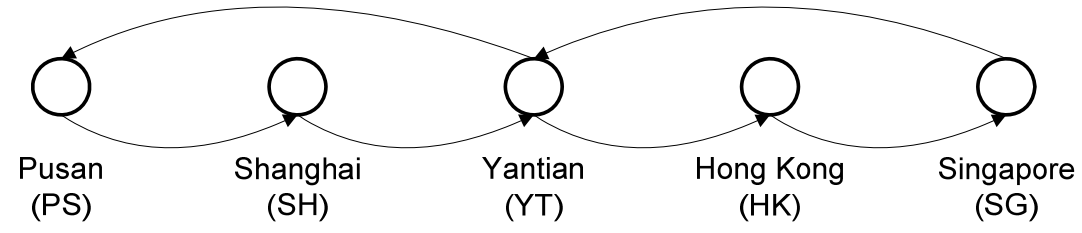

Figure 1 A shipping route

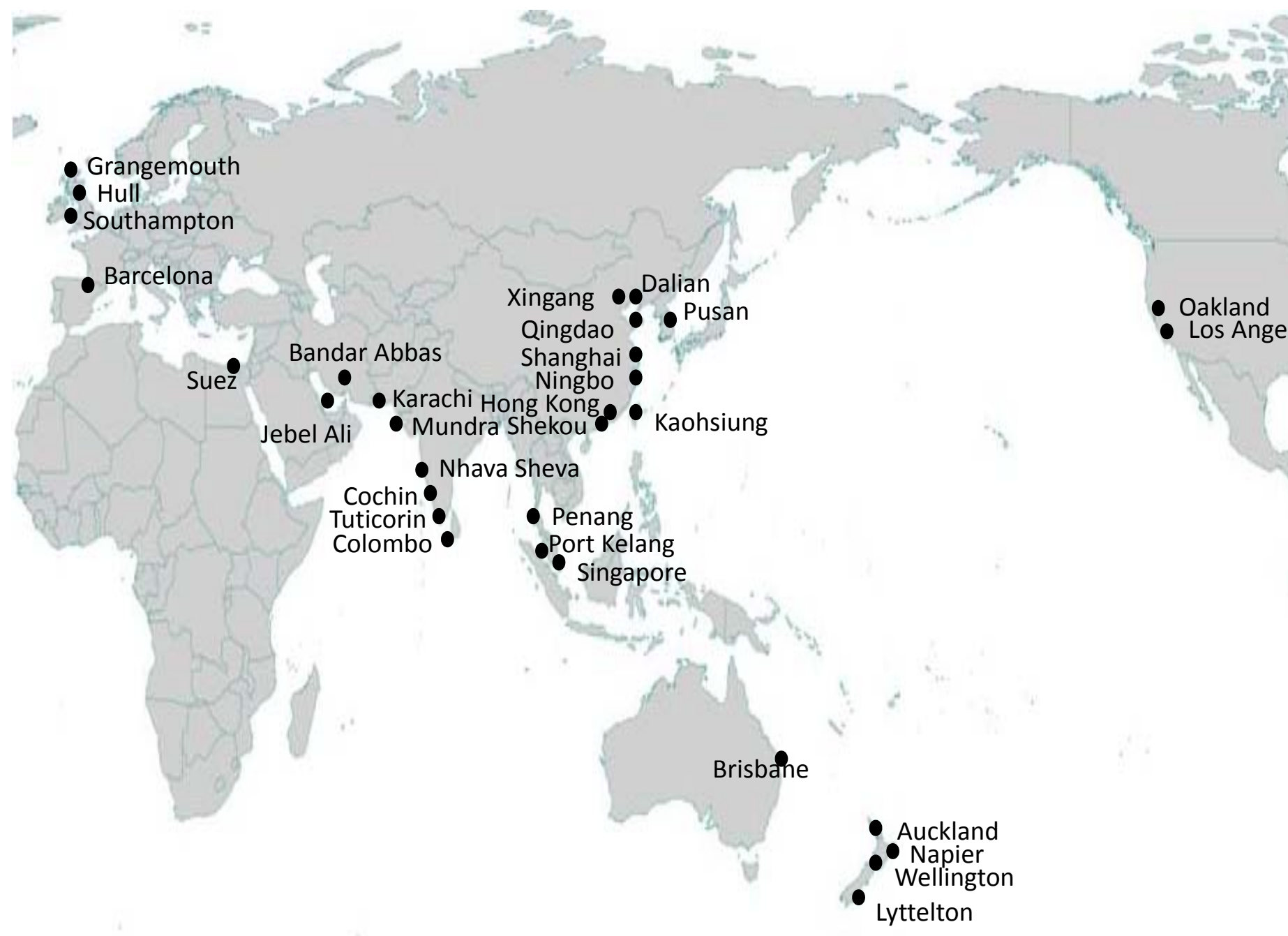

Figure 2 A real-world liner shipping network of OOCL(这个图似乎太大，页面放不下。) 


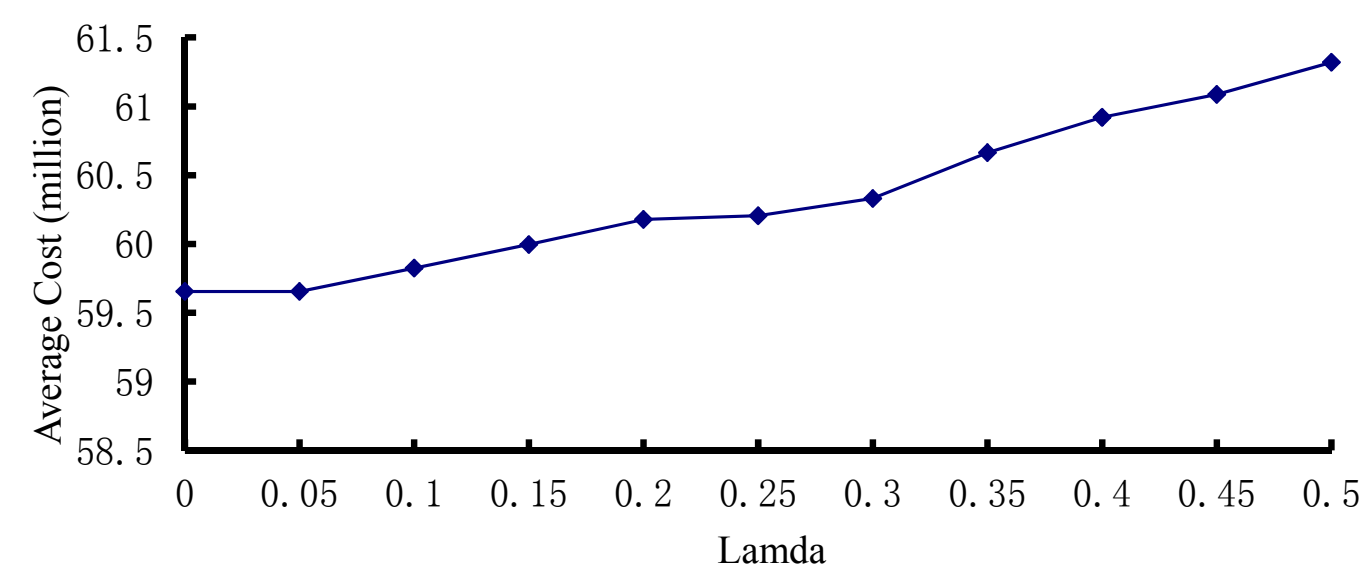

Figure 3 Average cost for different levels of variance

Table 1 Sensitivity analysis of SAA parameters

\begin{tabular}{ccccccccc}
\hline$\alpha$ & $\beta^{L}$ & $\beta^{U}$ & $\delta$ & $S$ & $S^{\prime}$ & Relative & $p^{S^{\prime}}\left(\hat{\mathbf{x}}^{*}\right)$ & $\begin{array}{c}\text { CPU } \\
\text { time (s) }\end{array}$ \\
\hline 0.05 & 0.08 & 0.030 & 0.10 & 1800 & 10000 & 0.85 & 0.0155 & 1695.52 \\
& 0.10 & 0.025 & 0.09 & 500 & 8000 & 0.73 & 0.0260 & 58.91 \\
& 0.12 & 0.020 & 0.08 & 300 & 6000 & 0.85 & 0.0162 & 30.99 \\
& 0.15 & 0.015 & 0.07 & 200 & 4000 & 1.24 & 0.0171 & 14.53 \\
& 0.20 & 0.010 & 0.06 & 100 & 2000 & 1.36 & 0.0092 & 2.66 \\
\hline 0.10 & 0.12 & 0.075 & 0.095 & 3000 & 10000 & 0.37 & 0.0795 & 587.03 \\
& 0.15 & 0.070 & 0.09 & 500 & 8000 & 0.76 & 0.0521 & 15.57 \\
& 0.18 & 0.060 & 0.08 & 200 & 6000 & 0.76 & 0.0468 & 8.23 \\
& 0.20 & 0.055 & 0.07 & 150 & 4000 & 0.76 & 0.0526 & 3.89 \\
& 0.25 & 0.050 & 0.06 & 100 & 2000 & 0.88 & 0.0415 & 3.63 \\
\hline 0.15 & 0.18 & 0.125 & 0.06 & 1600 & 10000 & 0.39 & 0.0755 & 65.94 \\
& 0.20 & 0.100 & 0.05 & 600 & 8000 & 0.39 & 0.1293 & 4.19 \\
& 0.25 & 0.075 & 0.04 & 200 & 6000 & 0.76 & 0.0494 & 0.60 \\
& 0.050 & 0.03 & 120 & 4000 & 0.94 & 0.0260 & 0.57 \\
& 0.025 & 0.02 & 100 & 2000 & 1.18 & 0.0271 & 0.47 \\
\hline
\end{tabular}


Table 2 Ports of call and distance for each leg of each shipping route

\begin{tabular}{|c|c|}
\hline Route & Port of calling (leg distance) \\
\hline \multirow[t]{3}{*}{ CCX } & Los Angeles $(360) \rightarrow$ Oakland $(4978) \rightarrow$ Pusan $(523) \rightarrow$ Dalian $(209) \rightarrow$ \\
\hline & Xingang $(408) \rightarrow$ Qingdao(390) $\rightarrow$ Ningbo $(111) \rightarrow$ Shanghai $(456) \rightarrow$ \\
\hline & Pusan $(5289) \rightarrow$ Los Angles \\
\hline \multirow[t]{3}{*}{ CPX } & Shanghai $(111) \rightarrow$ Ningbo $(740) \rightarrow \operatorname{Shekou}(1423) \rightarrow$ Singapore $(2881) \rightarrow$ \\
\hline & Karachi $(213) \rightarrow$ Mundra $(2474) \rightarrow$ Penang $(165) \rightarrow$ PortKelang $(198) \rightarrow$ \\
\hline & Singapore $(1422) \rightarrow$ Hong $\operatorname{Kong}(787) \rightarrow$ Shanghai \\
\hline \multirow[t]{3}{*}{ GIS } & Singapore $(198) \rightarrow$ Port Kelang $(2247) \rightarrow$ Nhava Sheva $(498) \rightarrow$ Karachi(713) \\
\hline & $\rightarrow$ Jebel Ali(152) $\rightarrow$ Bandar Abbas $(152) \rightarrow \operatorname{Jebel}$ Ali $(890) \rightarrow$ Mundra(915) \\
\hline & $\rightarrow$ Cochin $(1848) \rightarrow$ Singapore \\
\hline \multirow[t]{4}{*}{ IDX } & Colombo $(153) \rightarrow$ Tuticorin $(225) \rightarrow$ Cochin $(723) \rightarrow$ Nhava Sheva(372) $\rightarrow$ \\
\hline & $\operatorname{Mundra}(2809) \rightarrow \operatorname{Suez}(1673) \rightarrow$ Barcelona(3741) $\rightarrow$ NewYork(273) $\rightarrow$ \\
\hline & Norfolk $(402) \rightarrow$ Charleston $(4170) \rightarrow$ Barcelona $(1673) \rightarrow \operatorname{Suez}(3394) \rightarrow$ \\
\hline & Colombo \\
\hline \multirow[t]{3}{*}{ NCE } & New York $(273) \rightarrow \operatorname{Norfolk}(505) \rightarrow \operatorname{Savannah}(982) \rightarrow$ Panama $(13$ 831) $\rightarrow$ \\
\hline & $\operatorname{Pusan}(523) \rightarrow$ Dalian $(209) \rightarrow$ Xingang $(408) \rightarrow$ Qingdao $(390) \rightarrow$ \\
\hline & Ningbo(111) $\rightarrow$ Shanghai(13 565) $\rightarrow$ Panama(1359) $\rightarrow$ New York \\
\hline \multirow[t]{3}{*}{ NZX } & Singapore $(198) \rightarrow$ Port Kelang $(3880) \rightarrow$ Brisbane $(1303) \rightarrow$ Auckland(523) \\
\hline & $\rightarrow$ Napier(329) $\rightarrow$ Lyttelton $(175) \rightarrow$ Wellington $(1379) \rightarrow$ Brisbane $(3685) \rightarrow$ \\
\hline & Singapore \\
\hline \multirow[t]{3}{*}{ SCE } & New York $(273) \rightarrow$ Norfolk $(505) \rightarrow \operatorname{Savannah}(982) \rightarrow$ Panama $(12949) \rightarrow$ \\
\hline & Kaohsiung $(366) \rightarrow \operatorname{Shekou}(26) \rightarrow$ Hong $\operatorname{Kong}(12788) \rightarrow \operatorname{Panama}(1359) \rightarrow$ \\
\hline & New York \\
\hline UKX & Southampton $(315) \rightarrow \operatorname{Hull}(243) \rightarrow$ Grangemouth $(511) \rightarrow$ Southampton \\
\hline
\end{tabular}

Source: The port distances are from the website: http://www.searates.com/reference/portdistance/ 
Table 3 Ship data

\begin{tabular}{|c|c|c|c|c|c|}
\hline \multirow{2}{*}{ Item } & \multicolumn{5}{|c|}{ Ship types } \\
\hline & 1 & 2 & 3 & 4 & 5 \\
\hline Ship size (TEUs) ${ }^{a}$ & 2808 & 3218 & 4500 & 5714 & 8063 \\
\hline 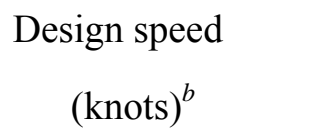 & 21.0 & 22.0 & 24.2 & 24.6 & 25.2 \\
\hline Daily cost $\left(10^{3} \$\right)^{c}$ & 19.8 & 22.5 & 30.9 & 38.8 & 54.2 \\
\hline $\begin{array}{c}\text { Chartering in rate } \\
\text { (million } \$)\end{array}$ & 2 & 2.6 & 3.5 & 4.7 & 6.0 \\
\hline$N_{k}^{\mathrm{MAX}}$ & 2 & 2 & 9 & 2 & 12 \\
\hline$N C I_{k}^{\mathrm{MAX}}$ & 5 & 5 & 5 & 3 & 3 \\
\hline
\end{tabular}

Source: ${ }^{a, b}$ From OOCL annual report, ${ }^{c}$ Calculation by Eq. (25). 